\title{
Output feedback controller design for a mixed traffic flow system moving in a loop
}

\section{Conference Paper}

\section{Author(s):}

Mousavi, Shimaossadat (D); Bahrami, Somayeh; Kouvelas, Anastasios (D)

Publication date:

2021

Permanent link:

https://doi.org/10.3929/ethz-b-000499111

Rights / license:

In Copyright - Non-Commercial Use Permitted

Originally published in:

https://doi.org/10.23919/acc50511.2021.9482943

Funding acknowledgement:

188622 - Real-time traffic estimation and control in a connected environment (SNF) 


\title{
Output Feedback Controller Design for a Mixed Traffic Flow System Moving in a Loop
}

\author{
Shima Sadat Mousavi ${ }^{\dagger}$, Somayeh Bahrami ${ }^{\ddagger}$, and Anastasios Kouvelas ${ }^{\dagger}$
}

\begin{abstract}
In this paper, we study a mixed traffic system moving along a single-lane ring road, that forms a platoon and includes a number of heterogeneous human-driven vehicles (HDVs) together with one connected and automated vehicle (CAV). The dynamics of HDVs are assumed to follow the optimal velocity model (OVM), and the acceleration of the single CAV is directly controlled by a dynamical output feedback controller. The ultimate goal of this work is to present a robust control strategy that can smoothen the traffic flow in the presence of undesired disturbances. A prerequisite for synthesizing a dynamical output controller is the stabilizability and detectability of the underlying system. Accordingly, a theoretical analysis is presented first to prove the stabilizability and detectability of the mixed traffic flow system. Then, an $H_{\infty}$ robust controller is designed, and its efficiency is illustrated through numerical simulations.
\end{abstract}

\section{INTRODUCTION}

In recent decades, the transition phase from using humandriven vehicles (HDVs) to fully connected and automated vehicles (CAVs) results in new challenges and creates a strong motivation to study the mixed traffic systems, that include both HDVs and CAVs [1]. More traditional methodologies for controlling traffic flow employ controllers and actuators at fixed locations, among which variable speed limits (VSLs) and ramp metering (RMs) can be mentioned [2]. However, the installation of these actuators is not costeffective and reduces the flexibility of the control system. On the other hand, the advent of CAVs as mobile actuatorsso-called Lagrangian actuators-pave the way for applying traffic flow control in a more effectivemanner. Nevertheless, in a mixed traffic system, new challenges are introduced that require further theoretical and experimental analyses.

For instance, in the study in [3], the outcome of a practical experiment on a single-lane ring road demonstrated that a platoon consisting only of HDVs has the potential to initiate stop-and-go waves. This phenomenon of instability has been studied in the literature from macroscopic [4], cellular automaton [5], and microscopic [6] point of view. In [7], a field experiment was conducted to show that utilizing a single $\mathrm{CAV}$ in a platoon on a circular roadway can dissipate the undesired waves. Moreover, in [8], through some theoretical analysis, the capability of a single CAV to control the traffic flow on a ring road was investigated.

Since a platoon of vehicles is connected and neighboring vehicles can communicate, a sparse number of CAVs-that

\footnotetext{
${ }^{\dagger}$ The authors are with the Institute for Transport Planning and Systems, ETH Zurich, Switzerland, \{shimaossadat.mousavi, anastasios.kouvelas\}eivt.baug.ethz.ch

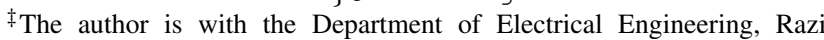
University, Kermanshah, Iran, s.bahrami@razi.ac.ir
}

act as mobile actuators-can influence the whole network and stabilize the traffic system. The notions of string and ring stability have been employed here for the stability analysis of interconnected vehicles on a string and on a ring roadway, respectively [9], [10]. In the same direction, and for mixed traffic systems, the string stability of a mixed platoon of infinite length has been analyzed in [11].

A fundamental network property that should be checked before designing a controller is its controllability or stabilizability [12]-[14]. Recently, some works in the literature have focused on providing a rigorous controllability and stabilizability analysis for ring-road mixed traffic systems with one single CAV. In [8], [15], it is assumed that all HDVs in the platoon are homogeneous, which is a strong assumption for practical scenarios. In fact, the problem where heterogeneity of HDVs is considered is closer to reality, but theoretically more challenging. In [16], [17], a controllability analysis for mixed traffic systems, including one CAV and a number of heterogeneous HDVs, is provided. It is stated that under a specific condition on some parameters of the model, all nonzero eigenvalues of the system are controllable; while there is one uncontrollable eigenvalue at origin.

In this paper, we consider a mixed traffic system with one CAV and numerous heterogeneous HDVs, moving along a ring road. The dynamics of HDVs are represented by the optimal velocity model, and the acceleration of the CAV is directly controlled. For this system, we first establish a stabilizability analysis in Section III. Since the goal is to synthesize an output feedback controller, the detectability analysis is also necessary, which is presented subsequently. In most of the works, proposing control strategies to stabilize a platoon on a ring road, the communication topology of the network which represents the capability of the CAV to receive information from its neighboring vehicles has been neglected [18]-[21], and it has been assumed that it is connected to any vehicle. Recently, in [16], the issue of limited communication has been considered and a structured optimal control has been proposed, which is computationally intractable [22] and results in a suboptimal solution.

In this work, we study a real-world scenario, and in order to deal with the topological communication constraints, we propose an output feedback controller in Section IV, that employs the information of a sparse set of vehicles in the control signal design. Moreover, unlike some existing control strategies, e.g., [23], [24], that aim to increase the efficiency of only the CAVs in the system, our method offers a robust $H_{\infty}$ controller, that dampens the disturbances appearing as nonlinear waves, and improves the performance in the 
behavior of the entire traffic network. Most importantly, the proposed controller leads to a global solution that has a better performance as compared to [16], [17], which is validated though numerical simulations.

The main contributions of this work compared to [17] can be summarised as: 1) Rather than the structural optimal control method, presented in [17], we propose a new solution based on synthesizing an output dynamic feedback controller to tackle the problem of communication constraints. To our best knowledge, this is the first time in the literature that an output dynamic controller is utilized for the control of a platoon. This approach in fact leads to an optimal solution, while structural control method in [17] results in a suboptimal one. 2) Unlike [17] that proves the stabilizability of a heterogeneous platoon under restrictive conditions on the value of system parameters, we demonstrate the system stabilizability for a general system with any arbitrary parameters. 3) In order to utilize a dynamic output controller, we show in this work that the system is also detectable.

\section{PRELIMinaries}

In this section, we present a model for a mixed traffic system on a single-lane ring road and formulate the problem.

We denote the set of real and complex numbers by $\mathbb{R}$ and $\mathbb{C}$, respectively. For $a \in \mathbb{C}, \operatorname{Re}(a)$ represents its real part. We denote the transpose of matrix $M$ by $M^{T}$. Also, $\operatorname{det}(M)$ represents its determinant. For a vector space $\mathcal{V}, \operatorname{dim}(\mathcal{V})$ indicates its dimension. The identity matrix is denoted by $I$, and its $j$-th column is designated by $e_{j}$.

\section{A. Modeling a mixed traffic system}

We consider a mixed traffic system that is a network of $n$ vehicles, including one CAV and $n-1$ HDVs, moving along a single-lane ring road with circumference $D$. In Fig. 1(a), a schematic diagram of this network is illustrated, where the red car denotes the $\mathrm{CAV}$, indexed with 1 , and all others are HDVs. The position and velocity of vehicle $i$ are denoted by $p_{i}$ and $v_{i}$, respectively, and we define as $s_{i}=p_{i-1}-p_{i}$ the spacing between vehicles $i$ and $i-1$.

There are different models in the literature to represent the car-following dynamics of human-driven vehicles (see e.g., [6], [25]). For instance, the optimal velocity model (OVM) [6] can be described as:

$$
\begin{aligned}
& \dot{s}_{i}(t)=v_{i-1}(t)-v_{i}(t) \\
& \dot{v}_{i}(t)=H_{i}\left(v_{i}(t), s_{i}(t), \dot{s}_{i}(t)\right),
\end{aligned}
$$

where $H_{i}(\cdot)$ is the acceleration of vehicle $i$, that is a nonlinear function of its velocity $v_{i}$, the spacing $s_{i}$, and the relative velocity $\dot{s}_{i}$. Note that unlike most of the works in literature (e.g., [1], [8], [18]), in this paper, HDVs are assumed to be heterogeneous, and thus, the dynamics of each vehicle $i$ is described by a distinct nonlinear function $H_{i}(\cdot)$. One can see that at the equilibrium point of dynamics (1), all vehicles have the same velocity $v^{*}$. Moreover, since we have $\dot{v}^{*}=0$, the spacing $s_{i}^{*}$ is computed by $0=H_{i}\left(v^{*}, s_{i}^{*}, 0\right)$.

Now, let us define the state error $x_{i}=\left[\begin{array}{ll}\bar{s}_{i} & \bar{v}_{i}\end{array}\right]^{T}=$ $\left[\begin{array}{ll}s_{i}-s_{i}^{*} & v_{i}-v^{*}\end{array}\right]^{T}$. Then, by linearization of (1) around the equilibrium point $\left(s_{i}^{*}, v^{*}\right)$, for $i=2, \ldots, n$, we derive a linear time-invariant (LTI) model for the HDV $i$ as:

$$
\begin{aligned}
& \dot{\bar{s}}_{i}(t)=\bar{v}_{i-1}(t)-\bar{v}_{i}(t) \\
& \dot{\bar{v}}_{i}(t)=\beta_{i 1} \bar{s}_{i}(t)-\beta_{i 2} \bar{v}_{i}(t)+\beta_{i 3} \bar{v}_{i-1},
\end{aligned}
$$

where

$$
\beta_{i 1}=\frac{\partial H_{i}}{\partial s_{i}}, \quad \beta_{i 2}=\frac{\partial H_{i}}{\partial \dot{s}_{i}}-\frac{\partial H_{i}}{\partial v_{i}}, \quad \beta_{i 3}=\frac{\partial H_{i}}{\partial \dot{s}_{i}},
$$

computed at the equilibrium point. Due to some physical constraints imposed by the behavior of HDVs in practice [8], one should consider

$$
\beta_{i 1}>0, \quad \beta_{i 2}>0, \quad \beta_{i 3}>0 .
$$

The dynamics of the single CAV whose acceleration can be directly controlled are also given by:

$$
\begin{aligned}
& \dot{\bar{s}}_{1}(t)=\bar{v}_{n}(t)-\bar{v}_{1}(t) \\
& \dot{\bar{v}}_{1}(t)=u(t)
\end{aligned}
$$

where $u(t) \in \mathbb{R}$ is the control signal. Now, by defining the aggregated vector of states of all vehicles as $x=$ $\left[\begin{array}{llll}x_{1}^{T} & x_{2}^{T} & \ldots & x_{n}^{T}\end{array}\right]^{T} \in \mathbb{R}^{2 n}$, we derive the following LTI dynamics for the overall system:

$$
\dot{x}(t)=A x(t)+B u(t)
$$

where

$$
A=\left[\begin{array}{cccccc}
J_{1} & 0 & \ldots & \ldots & 0 & J_{2} \\
A_{21} & A_{22} & 0 & \ldots & \ldots & 0 \\
0 & A_{31} & A_{32} & 0 & \ldots & 0 \\
\vdots & \ddots & \ddots & \ddots & \ddots & \vdots \\
0 & \ldots & \ldots & 0 & A_{n 1} & A_{n 2}
\end{array}\right], \quad B=\left[\begin{array}{c}
B_{1} \\
B_{2} \\
B_{2} \\
\vdots \\
B_{2}
\end{array}\right]
$$

with

$$
J_{1}=\left[\begin{array}{cc}
0 & -1 \\
0 & 0
\end{array}\right], \quad J_{2}=\left[\begin{array}{ll}
0 & 1 \\
0 & 0
\end{array}\right], \quad B_{1}=\left[\begin{array}{l}
0 \\
1
\end{array}\right], \quad B_{2}=\left[\begin{array}{l}
0 \\
0
\end{array}\right] .
$$

Moreover, for $i=2, \ldots, n$, we have

$$
A_{i 1}=\left[\begin{array}{cc}
0 & 1 \\
0 & \beta_{i 3}
\end{array}\right], \quad A_{i 2}=\left[\begin{array}{cc}
0 & -1 \\
\beta_{i 1} & -\beta_{i 2}
\end{array}\right]
$$

Finally, we note that the CAV can receive the state information associated with only a number of HDVs, due to its communications constraints. For example, in Fig. 1(a) and (b), the green links show HDVs whose information is available to the CAV. In this direction, an output vector

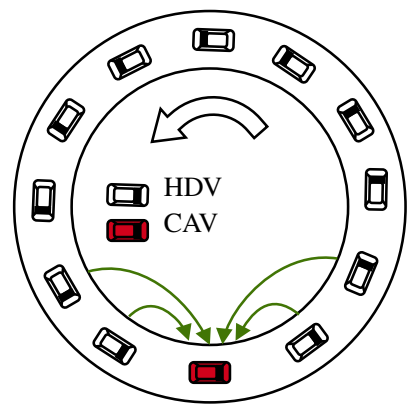

(a)

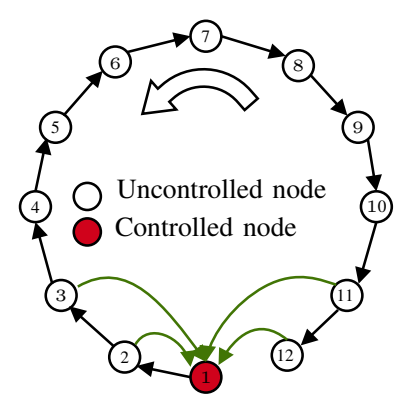

(b)
Fig. 1. (a) Schematic of Mixed traffic system; (b) the corresponding graph. 
$y(t) \in \mathbb{R}^{2 m}$ is defined, that includes the states of the CAV together with the states of all HDVs whose information is accessible by the CAV. For $k=1, \ldots, m$, let $j_{k}$ be the index of vehicle whose state information, i.e. $\bar{s}_{j_{k}}$ and $\bar{v}_{j_{k}}$, can be directly measured and observed. Consequently, we have:

$$
y(t)=C x(t),
$$

where $C=\left[\begin{array}{lllll}e_{\left(2 * j_{1}-1\right)} & e_{2 * j_{1}} & \ldots & e_{\left(2 * j_{m}-1\right)} & e_{2 * j_{m}}\end{array}\right]^{T}$. Note that we can have $j_{1}=1$, since the CAV has access to its own information.

\section{B. Disturbances and performance outputs}

In this paper, we aim to design an output controller for the mixed traffic system (3) to dissipate undesired perturbations. In fact, the perturbations may appear due to lane changes or merges or the stochastic behavior of HDVs in ring roads with no bottlenecks [3], [17].

Perturbations are modeled as disturbance signals added to the acceleration of each vehicle. Thus, one can define a vector of disturbances as $d=\left[\begin{array}{lll}d_{1}(t) & \ldots & d_{n}(t)\end{array}\right]^{T} \in \mathbb{R}^{n}$. In addition, a vector of output signals related to the system performance can be defined as $z(t)=\left[\begin{array}{llllll}\gamma_{s} \bar{s}_{1}(t) & \gamma_{v} \bar{v}_{1} & \ldots & \gamma_{s} \bar{s}_{n}(t) & \gamma_{v} \bar{v}_{n} & \gamma_{u} u\end{array}\right]^{T}$, with $\gamma_{s}, \gamma_{v}, \gamma_{u}>0$ denoting the penalties associated with spacing error, velocity deviation, and control energy, respectively. Then, the dynamics of the mixed traffic system in the presence of disturbances can be written as

$$
\begin{aligned}
\dot{x}(t) & =A x(t)+B u(t)+B_{d} d(t) \\
z(t) & =C_{z} x(t)+D_{z} u(t) \\
y(t) & =C x(t)
\end{aligned},
$$

where $C_{z}=\left[\begin{array}{ll}P^{\frac{1}{2}} & 0\end{array}\right]^{T}$ and $P^{\frac{1}{2}}=\operatorname{diag}\left(\gamma_{s}, \gamma_{v}, \ldots, \gamma_{s}, \gamma_{v}\right)$. Moreover, $D_{z}=\left[\begin{array}{ll}0 & Q^{\frac{1}{2}}\end{array}\right]^{T}$, with $Q^{\frac{1}{2}}=\gamma_{u}$. By considering $b_{d}=[0,1]^{T}$, the matrix $B_{d}$ is defined as

$$
B_{d}=\left[\begin{array}{cccc}
b_{d} & 0 & \ldots & 0 \\
0 & b_{d} & \ldots & \vdots \\
\vdots & \ddots & \ddots & 0 \\
0 & \ldots & 0 & b_{d}
\end{array}\right] .
$$

\section{Problem formulation}

Let $T_{z d}$ be the closed-loop transfer function from the disturbance $d$ to the performance output $z$. Our goal is to design a dynamical output feedback controller for plant (6), minimizing the influence of $d$ on $z$. More formally, we compute an output feedback controller with the dynamics

$$
\begin{aligned}
\dot{x}_{k}(t) & =A_{k} x_{k}(t)+B_{k} y(t), \\
u(t) & =C_{k} x_{k}(t)
\end{aligned}
$$

that minimizes $\gamma>0$ such that

$$
\left\|T_{z d}\right\|_{\infty}=\max _{d(t) \neq 0} \frac{\|z(t)\|_{2}}{\|d(t)\|_{2}}<\gamma .
$$

Note that $\left\|T_{z d}\right\|_{\infty}$ denotes the $H_{\infty}$ norm of $T_{z d}$, which measures the largest input-output gain for energy or RMS input signals [26]. We also have $A_{k} \in \mathbb{R}^{2 n \times 2 n}, B_{k} \in$ $\mathbb{R}^{2 n \times 2 m}$ and $C_{k} \in \mathbb{R}^{1 \times 2 m}$.
In order to ensure the existence of an output feedback controller, based on the separation principle [27], we should first prove the stabilizability of pair $(A, B)$ and detectability of pair $(A, C)$ in (6). Pair $(A, B)$ is stabilizable if the uncontrollable modes are all stable. Similarly, pair $(A, C)$ is detectable if the unobservable modes are all stable. We can use Popov-Belevitch-Hautus (PBH) test for checking the controllability and observability of a specific eigenvalue.

Proposition 1 ([28]): An eigenvalue $\lambda$ of $A$ for a pair $(A, B)$ (resp., $(A, C))$ is controllable (resp., observable) if and only if for all nonzero $\rho$ for which $\rho^{T} A=\lambda \rho^{T}$ (resp., $A \rho=\lambda \rho$ ), $\rho^{T} B \neq 0$ (resp., $C \rho \neq 0$ ).

We dedicate the next section to analyze the stabilizability and detectability of the mixed traffic system (6), and subsequently, we design the dynamical controller.

Remark 1: In [16], [17], a structured state feedback controller has been designed to minimize the $H_{2}$ norm of $T_{z d}$. In that approach, a static controller is considered, but since only the state information of a limited number of vehicles is available, some entries of the controller gain are required to be zero, which results in a sub-optimal controller. In the current work, we synthesize a dynamical controller with two degrees of freedom, which while on the one hand considers the limited communication ability of the CAV, on the other hand, it can lead to a global and more efficient solution.

\section{Stabilizability AND Detectability}

In this section, the stabilizability and detectability of dynamical system (6) are discussed.

Proposition 2: The pair $(A, B)$ in (6) has only one uncontrollable eigenvalue at the origin.

Proof: Let $\rho=\left[\rho_{1}^{T}, \ldots, \rho_{n}^{T}\right]^{T} \in \mathbb{R}^{2 n}$, where $\rho_{i}=$ $\left[\rho_{i 1}, \rho_{i 2}\right]^{T}$. Considering the expression of $A$ in (4), equation $\rho^{T} A=0$ leads to

$$
\begin{aligned}
& \rho_{i}^{T} A_{i 2}+\rho_{i+1}^{T} A_{(i+1) 1}=0, \quad i=2, \ldots, n-1 \\
& \rho_{1}^{T} J_{1}+\rho_{2}^{T} A_{21}=0 \\
& \rho_{1}^{T} J_{2}+\rho_{n}^{T} A_{n 2}=0
\end{aligned} .
$$

Now, from (8), one can derive

$$
\begin{aligned}
\rho_{i 2} & =0, & & i=2, \ldots, n \\
\rho_{i 1} & =\rho_{(i+1) 1}, & & i=1, \ldots, n-1 .
\end{aligned}
$$

Thus, a left eigenvector $\rho$ of $A$ associated with $\lambda=0$ can be written as $\rho=[\alpha, \beta, \alpha, 0, \ldots, \alpha, 0]^{T}$. Now assume that $\lambda=0$ is an uncontrollable eigenvalue. Thus, from Proposition 1 , one can see that $\rho^{T} B=0$, which essentially means $\rho_{12}=0$. Now, define $V_{u}=\left\{\rho \in \mathbb{R}^{2 n}\right.$ : $\left.\rho^{T} A=0, \rho^{T} B=0\right\}$. Then, one can see that $V_{u}=$ $\left\{\rho \in \mathbb{R}^{2 n}: \rho=[\alpha, 0, \alpha, 0, \ldots, \alpha, 0]^{T}\right.$, for some $\left.\alpha \neq 0\right\}$. Thus, $\operatorname{dim}\left(V_{u}\right)=1$, which implies that there is only one uncontrollable eigenvalue of $A$ at the origin.

Next, it suffices to show that all unstable eigenvalues of $A$ in (6), if any, are controllable.

Lemma 1: Let $\lambda \in \mathbb{C}$ with $\operatorname{Re}(\lambda)>0$, and for $i=$ $2, \ldots, n$, let $A_{i 2}$ be defined as (5). Then, matrix $\left(\lambda I-A_{i 2}\right)$ is nonsingular. 
Proof: For $i=2, \ldots, n$, define $\mathcal{S}_{i}(\lambda)=\operatorname{det}(\lambda I-$ $\left.A_{i 2}\right)=\lambda^{2}+\beta_{i 2} \lambda+\beta_{i 1}$. Based on this equation, the sum of eigenvalues of $A_{i 2}$ equals $-\beta_{i 2}$, and their product is $\beta_{i 1}$. If the roots of $\mathcal{S}_{i}(\lambda)$ are real, then, since $\beta_{i 1}, \beta_{i 2}>0$ from (2), both the roots are negative. Moreover, if the roots are complex and are written as $a+i b$ and $a-i b$, then since $2 a=-\beta_{i 2}$, we have $a<0$. Therefore, because we have $\operatorname{Re}(\lambda)>0$, one can conclude that $\mathcal{S}_{i}(\lambda) \neq 0$, implying that for $i=2, \ldots, n,\left(\lambda I-A_{i 2}\right)$ is nonsingular.

Theorem 1: The pair $(A, B)$ associated with the mixed traffic system described in (6) is stabilizable.

Proof: Let $\lambda$ be an eigenvalue of $A$ with $\operatorname{Re}(\lambda)>0$, and let $\rho=\left[\rho_{1}^{T}, \ldots, \rho_{n}^{T}\right]^{T} \in \mathbb{R}^{2 n}$ be its nonzero left eigenvector, where $\rho_{i}=\left[\rho_{i 1}, \rho_{i 2}\right]^{T}$. Define $A_{11}=J_{2}$ and $A_{12}=J_{1}$. Then, for $i=1, \ldots, n$, equation $\rho^{T} A=\lambda \rho^{T}$ implies that

$$
\rho_{i}^{T}\left(\lambda I-A_{i 2}\right)=\rho_{i+1}^{T} A_{(i+1) 1} .
$$

Now, one can see that $\operatorname{det}\left(\lambda I-A_{12}\right)=\lambda^{2}$, which is nonzero, since $\lambda \neq 0$. Moreover, since $\operatorname{Re}(\lambda)>0$, Lemma 1 implies that $\left(\lambda I-A_{i 2}\right)$ is nonsingular. Accordingly, for $i=1, \ldots, n$ one can rewrite (9) as

$$
\rho_{i}^{T}=\rho_{i+1}^{T} A_{(i+1) 1}\left(\lambda I-A_{i 2}\right)^{-1} .
$$

Let $L_{i}=\left(\lambda I-A_{i 2}\right)^{-1}$. Now, by recursively employing equation (10) for $i=1, \ldots, n$, we can derive

$$
\begin{aligned}
\rho_{1}^{T} & =\rho_{2}^{T} A_{21} L_{1} \\
& =\rho_{3}^{T} A_{31} L_{2} A_{21} L_{1} \\
& =\ldots \\
& =\rho_{n}^{T} A_{n 1} L_{n-1} A_{(n-1) 1} \ldots L_{2} A_{21} L_{1} \\
& =\rho_{1}^{T} A_{11}\left(L_{n} A_{n 1}\right) \ldots\left(L_{2} A_{21}\right) L_{1} .
\end{aligned}
$$

Now, note that we have

$$
L_{i} A_{i 1}=\frac{1}{s_{1}^{i}}\left[\begin{array}{ll}
0 & s_{2}^{i} \\
0 & s_{3}^{i}
\end{array}\right], \quad i=2, \ldots, n,
$$

where $s_{1}^{i}=\lambda^{2}+\beta_{i 2} \lambda+\beta_{i 1}, s_{2}^{i}=\lambda+\beta_{i 2}-\beta_{i 3}$, and $s_{3}^{i}=$ $\beta_{i 3} \lambda+\beta_{i 1}$. As shown before, $s_{1}^{i} \neq 0$. Now, we substitute (12) into (11) and obtain

$$
\left[\begin{array}{ll}
\rho_{11} & \rho_{12}
\end{array}\right]=\frac{\Pi_{i=2}^{n} s_{3}^{i}}{\lambda \Pi_{i=2}^{n} s_{1}^{i}}\left[\begin{array}{ll}
\rho_{11} & \rho_{12}
\end{array}\right]\left[\begin{array}{ll}
0 & 1 \\
0 & 0
\end{array}\right],
$$

that leads to $\rho_{11}=0$ and $\rho_{12}=\frac{\Pi_{i=2}^{n} s_{3}^{i}}{\lambda \Pi_{i=2} s_{1}^{i}} \rho_{11}=0$. Thus, $\rho_{1}=$ 0 . Now, by recursively applying (10) for $i=n, n-1, \ldots, 2$, one can conclude that $\rho_{i}=0$. Therefore, we have $\rho=0$ that contradicts the assumption. Thus, $A$ has no eigenvalue which lies on the right half-plane. In addition, from Proposition 2, there is only one uncontrollable eigenvalue at origin, and hence the system is stabilizable.

Remark 2: In [17], by considering a certain constraint on the values of $\beta_{i 1}, \beta_{i 2}$, and $\beta_{i 3}$, for $i=1, \ldots, n$, it has been stated that all the nonzero eigenvalues are controllable. It is also shown that there is only one uncontrollable eigenvalue at origin. On the other hand, in this paper, the stabilizability of a mixed traffic system with heterogeneous HDVs is proved for the most general case and without assuming any constraint.
Now, the detectability of the mixed traffic system is studied, and we show that by observing only the states of the single CAV, the detectability can be ensured.

Proposition 3: The zero eigenvalue of the mixed traffic system described in (6) is observable even if the CAV has access to only its own states.

Proof: Let $C=\left[\begin{array}{ll}e_{1} & e_{2}\end{array}\right]^{T}$, and assume that $\lambda=0$ is not observable. Then, from Proposition $1, A$ has a nonzero right eigenvector $\rho$, where we have both $A \rho=0$ and $C \rho=0$. Let $\rho=\left[\rho_{1}^{T}, \ldots, \rho_{n}^{T}\right]^{T} \in \mathbb{R}^{2 n}$, where $\rho_{i}=\left[\rho_{i 1}, \rho_{i 2}\right]^{T}$. From equation $A \rho=0$, one can write:

$$
\begin{array}{ll}
\rho_{i 2}-\rho_{(i+1) 2}=0, & i=1, \ldots, n \\
\beta_{i 3} \rho_{(i-1) 2}+\beta_{i 1} \rho_{i 1}-\beta_{i 2} \rho_{i 2}=0, & i=2, \ldots, n
\end{array} .
$$

Moreover, from $C \rho=0$, one can conclude that $\rho_{11}=$ $\rho_{12}=0$. Thus, (13) leads to $\rho=0$, which is a contradiction. Therefore, the zero eigenvalue is observable.

Theorem 2: The mixed traffic system (6) is detectable even if only the states of the CAV are directly observed.

Proof: Let $C=\left[\begin{array}{ll}e_{1} & e_{2}\end{array}\right]^{T}$. Assume the system is not detectable. Then, $A$ has an eigenvalue $\lambda$ on the right halfplane with a nonzero right eigenvector $\rho$ such that $C \rho=0$. Denote $\rho=\left[\rho_{1}^{T}, \ldots, \rho_{n}^{T}\right]^{T} \in \mathbb{R}^{2 n}$, where $\rho_{i}=\left[\rho_{i 1}, \rho_{i 2}\right]^{T}$. Then, $\rho_{11}=\rho_{12}=0$. Define $A_{11}=J_{2}$ and $A_{12}=J_{1}$. Now, from equation $A \rho=\lambda \rho$, for $i=1, \ldots, n$, one can write:

$$
\left(\lambda I-A_{i 2}\right) \rho_{i}=A_{i 1} \rho_{i-1} .
$$

Since $\operatorname{Re}(\lambda)>0$, from Lemma 1 , for $i=2, \ldots, n,(\lambda I-$ $\left.A_{i 2}\right)$ is invertible. Moreover, $\operatorname{det}\left(\lambda I-A_{12}\right)=\lambda^{2} \neq 0$. Let $L_{i}=\left(\lambda I-A_{i 2}\right)^{-1}$. Then, for $i=1, \ldots, n$, we can write:

$$
\rho_{i}=L_{i} A_{i 1} \rho_{i-1} \text {. }
$$

Now, since $\rho_{1}=0$, recursively using equation (14) for $i=2, \ldots, n$ leads to $\rho=0$, contradicting the assumption. In addition, from Proposition 4, the zero eigenvalue is observable. Thus, the system is detectable.

\section{Robust CONTROLlER SyNTHESIS}

In this section, we propose an output feedback controller that stabilizes the mixed traffic system (6) and minimizes the impact of disturbance on the performance output.

As formulated in Section II-B, by defining the performance output $z$, the state-space realization of the open-loop system in the presence of the disturbances is

$$
M:\left[\begin{array}{c|cc}
A & B & B_{d} \\
\hline C_{z} & D_{z} & 0 \\
C & 0 & 0
\end{array}\right] .
$$

By considering an output controller as

$$
K:\left[\begin{array}{c|c}
A_{k} & B_{k} \\
\hline C_{k} & 0
\end{array}\right],
$$

the state-space equations of the closed-loop system become

$$
\begin{aligned}
{\left[\begin{array}{c}
\dot{x}(t) \\
\dot{x}_{k}(t)
\end{array}\right] } & =\left[\begin{array}{cc}
A & B C_{k} \\
B_{k} C & A_{k}
\end{array}\right]\left[\begin{array}{c}
x(t) \\
x_{k}(t)
\end{array}\right]+\left[\begin{array}{c}
B_{d} \\
0
\end{array}\right] d(t) \\
z(t) & =\left[\begin{array}{ll}
C_{z} & D_{z} C_{k}
\end{array}\right]\left[\begin{array}{c}
x(t) \\
x_{k}(t)
\end{array}\right] .
\end{aligned}
$$


Therefore

$$
T_{z d}:\left[\begin{array}{cc|c}
A & B C_{k} & B_{d} \\
B_{k} C & A_{k} & 0 \\
\hline C_{z} & D_{z} C_{k} & 0
\end{array}\right]=\left[\begin{array}{c|c}
\tilde{A} & \tilde{B} \\
\hline \tilde{C} & 0
\end{array}\right] .
$$

Now, based on the bounded real lemma (BRL) [29], we can write the performance criteria in (7) as a linear matrix inequality (LMI). In fact, $\left\|T_{z d}\right\|_{\infty}$ is smaller than $\gamma$ if and only if there exists a positive definite matrix $P \succ 0$, together with matrices $A_{k}, B_{k}$, and $C_{k}$, satisfying

$$
\left[\begin{array}{ccc}
\tilde{A}^{T} P+P \tilde{A} & P \tilde{B} & \tilde{C}^{T} \\
\tilde{B}^{T} P & -I & 0 \\
\tilde{C} & 0 & -\gamma^{2} I
\end{array}\right] \prec 0 .
$$

In order to compute the controller parameters $A_{k}, B_{k}$, and $C_{k}$, we apply a method that has been presented in details in [26]. We provide here a summarized description.

Matrix $P$ can be partitioned in the following form

$$
P=\left[\begin{array}{cc}
Y & N \\
N^{T} & *
\end{array}\right], \quad P^{-1}=\left[\begin{array}{cc}
X & M \\
M^{T} & *
\end{array}\right] .
$$

Note that, equation $P P^{-1}=I$ yields $N M^{T}=I-Y X$. Now, let us define a new set of variables as

$$
\begin{aligned}
& \hat{A}=N A_{k} M^{T}+N B_{k} C X+Y B C_{k} M^{T}+Y A X \\
& \hat{B}=N B_{k} \\
& \hat{C}=C_{k} M^{T} .
\end{aligned}
$$

It has been shown that the $H_{\infty}$ problem in (7) has an optimal solution if one can find matrices $\hat{A}, \hat{B}$, and $\hat{C}$, and positive definite matrices $X \succ 0, Y \succ 0$, such that the LMIs described in (17) are satisfied.

If the optimization problem (17) is feasible, and $\eta, \hat{A}, \hat{B}$, $\hat{C}, X$, and $Y$ can be found, we can solve the matrix equation $N M^{T}=I-Y X$ for the non-singular matrices $M$ and $N$. Also, $A_{k}, B_{k}$, and $C_{k}$ can be obtained based on (16) as:

$$
\begin{aligned}
& A_{k}=N^{-1}\left(\hat{A}-N B_{k} C X-Y B C_{k} M^{T}-Y A X\right) M^{-T} \\
& B_{k}=N^{-1} \hat{B} \\
& C_{k}=\hat{C} M^{-T} .
\end{aligned}
$$

\section{A. Simulation results}

Here, the efficiency of the proposed control strategy is validated through numerical simulations. For a better comparison of the results, we simulate in MATLAB an experimental setup that is quite similar to the one considered in [16], [17].

A ring road with a circumference $D=400 \mathrm{~m}$ and 20 vehicles has been considered, where the single CAV has access to state information of five HDVs ahead and five HDVs behind. The parameters $\gamma_{s}, \gamma_{v}$, and $\gamma_{u}$, appearing in the equation of performance output $z$ in (6), are set as $\gamma_{s}=0.03, \gamma_{v}=0.15$, and $\gamma_{u}=1$. The nonlinear function $H(\cdot)$ in (1) that describes the acceleration function of HDVs is written as $H_{i}(\cdot)=\alpha_{i}\left(Q_{i}\left(s_{i}(t)\right)-v_{i}(t)\right)+\theta_{i} \dot{s}_{i}(t)$, where $\alpha_{i}=0.6+U[-0.1,0.1]$ and $\theta_{i}=0.9+U[-0.1,0.1] . U[\cdot]$ represents the uniform distribution function, and $Q_{i}(\cdot)$ is a piecewise function described as

$$
Q_{i}(s)= \begin{cases}0, & s \leq s_{i, s t}, \\ h_{i, v}(s), & s_{i, s t}<s<s_{i, g o}, \\ v_{i, \max }, & s \geq s_{i, g o},\end{cases}
$$

where $s_{i, s t}=5, s_{i, g o}=35+U[-5,5], v_{i, \max }=30$, and $h_{i, v}$ is a nonlinear function [18] chosen as

$$
h_{i, v}(s)=\frac{v_{i, \max }}{2}\left(1-\cos \left(\pi \frac{s-s_{i, s t}}{s_{i, g o}-s_{i, s t}}\right)\right) .
$$

To prevent collisions, every vehicle is also assumed to be equipped with an automatic braking system, described as

$$
\dot{v}(t)=a_{\min }, \quad \text { if } \quad \frac{v_{i}^{2}(t)-v_{i-1}^{2}(t)}{2 s_{i}(t)} \geq\left|a_{\min }\right|,
$$

where $a_{\min }=-5 \mathrm{~m} / \mathrm{s}^{2}$. Moreover, let $a_{\max }=2 \mathrm{~m} / \mathrm{s}^{2}$.

Now, assume that all vehicles are randomly distributed along the ring road and start their movement with initial velocity $v_{i}(0)=15+U[-4,4] \mathrm{m} / \mathrm{s}$. Now, consider the case where all vehicles of the mixed traffic system are HDVs. In Fig. 2(a), it can be seen that if multiple perturbations occur in this system, they are amplified over time and generate an unstable nonlinear wave moving upstream the traffic flow. Next, assume that one of the vehicles is a CAV that is controlled by the proposed strategy. In this case, from Fig. 2(b), we observe that the perturbations are dampened, and the traffic flow is stabilized to the average velocity. It should be noted that employing the proposed controller in this paper leads to a higher convergence rate of velocity to the average value as compared to the structured optimal control method in [16]. In [16], the system is stabilized to the average velocity within $40 \mathrm{~s}$, while here, the convergence occurs within 10 $\mathrm{s}$. We can also change the desired equilibrium velocity to 16 and $14 \mathrm{~m} / \mathrm{s}$, respectively. Considering Fig. 2(c)-(d), one can see that in these cases, the single CAV is still capable of stabilizing traffic flow and steering it towards the new equilibrium points, which verifies the stabilizability of mixed traffic system with only one CAV.

\section{CONCLUSION}

In this work, the stabilizability and detectability of a mixed traffic ring-road system was analyzed. Furthermore, by considering limited communication ability of the CAV to receive state information from its neighboring vehicles, an $H_{\infty}$ dynamic output feedback controller was designed, that can provably smooth traffic flow even in the presences of disturbances. The effectiveness of the proposed method to achieve traffic flow stability has been verified through numerical simulation experiments.

\section{ACKNOWLEDGEMENT}

This work was supported in part by the Swiss National Science Foundation (SNSF) under the project RECCE "Realtime traffic estimation and control in a connected environment", contract No. 200021-188622. 


$$
\min _{\eta, X, Y, \hat{A}, \hat{B}, \hat{C}} \eta
$$

subject to:

$$
\begin{aligned}
& {\left[\begin{array}{cc}
X & I \\
I & Y
\end{array}\right]>0,} \\
& {\left[\begin{array}{cccc}
A X+X A^{T}+B \hat{C}+\hat{C}^{T} B^{T} & \hat{A}^{T}+A & B_{d}^{T} & X C_{z}^{T}+\hat{C}^{T} D_{z}^{T} \\
* & A^{T} Y+Y A+\hat{B} C+C^{T} \hat{B}^{T} & Y B_{d} & C_{z}^{T} \\
* & * & -I & 0 \\
* & * & * & -\eta I
\end{array}\right]<0 .}
\end{aligned}
$$
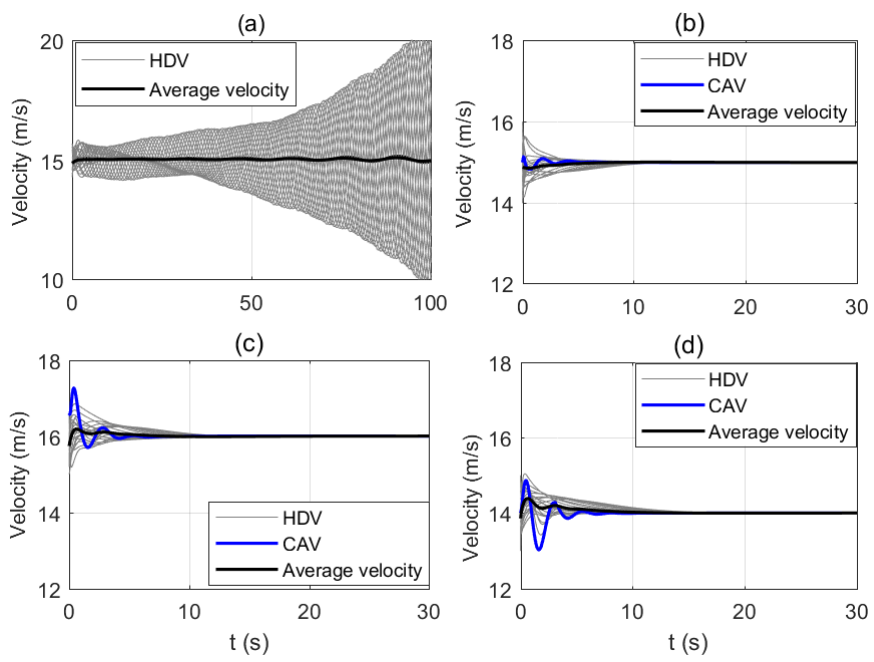

Fig. 2. (a) Velocity profile of vehicles when they are all HDVs; (b)(d) velocity profile of vehicles when there is one CAV under the proposed control, and $v^{*}$ is 15,16 , and 14 for (b), (c), and (d), respectively.

\section{REFERENCES}

[1] V. Giammarino, M. Lv, S. Baldi, P. Frasca, and M. L. Delle Monache, "On a weaker notion of ring stability for mixed traffic with humandriven and autonomous vehicles," in Proc. 58th IEEE Conf. on Decision and Control, 2019, pp. 335-340.

[2] I. Papamichail, K. Kampitaki, M. Papageorgiou, and A. Messmer, "Integrated ramp metering and variable speed limit control of motorway traffic flow," IFAC Proc. Vol., vol. 41, no. 2, pp. 14 084-14 089, 2008.

[3] Y. Sugiyama, M. Fukui, M. Kikuchi, K. Hasebe, A. Nakayama, K. Nishinari, S.-i. Tadaki, and S. Yukawa, "Traffic jams without bottlenecks-experimental evidence for the physical mechanism of the formation of a jam," New J. Phys., vol. 10, no. 3, p. 033001, 2008.

[4] M. R. Flynn, A. R. Kasimov, J.-C. Nave, R. R. Rosales, and B. Seibold, "Self-sustained nonlinear waves in traffic flow," Phys. Rev. E, vol. 79, no. 5 , p. $056113,2009$.

[5] K. Nagel and M. Schreckenberg, "A cellular automaton model for freeway traffic," J. Phys. I France, vol. 2, no. 12, pp. 2221-2229, 1992.

[6] M. Bando, K. Hasebe, A. Nakayama, A. Shibata, and Y. Sugiyama, "Dynamical model of traffic congestion and numerical simulation," Phys. Rev. E, vol. 51, no. 2, p. 1035, 1995.

[7] R. E. Stern, S. Cui, M. L. Delle Monache, R. Bhadani, M. Bunting, M. Churchill, N. Hamilton, H. Pohlmann, F. Wu, B. Piccoli, et al., "Dissipation of stop-and-go waves via control of autonomous vehicles: Field experiments," Transp. Res. Part C: Emerging Tech., vol. 89, pp. 205-221, 2018.

[8] S. Cui, B. Seibold, R. Stern, and D. B. Work, "Stabilizing traffic flow via a single autonomous vehicle: Possibilities and limitations," in IEEE Intell. Vehicles Sympos., 2017, pp. 1336-1341.

[9] D. Swaroop and J. K. Hedrick, "String stability of interconnected systems," IEEE Trans. Automat. Contr., vol. 41, no. 3, pp. 349-357, 1996.
[10] J. Ploeg, N. Van De Wouw, and H. Nijmeijer, "Lp string stability of cascaded systems: Application to vehicle platooning," IEEE Trans. Contr. Syst. Tech., vol. 22, no. 2, pp. 786-793, 2013.

[11] A. Talebpour and H. S. Mahmassani, "Influence of connected and autonomous vehicles on traffic flow stability and throughput," Transp. Res. Part C: Emerg. Tech., vol. 71, pp. 143-163, 2016.

[12] S. S. Mousavi, M. Haeri, and M. Mesbahi, "On the structural and strong structural controllability of undirected networks," IEEE Trans. Automat. Contr., vol. 63, no. 7, pp. 2234-2241, 2018.

[13] S. S. Mousavi, M. Haeri, and M. Mesbahi, "Strong structural controllability of networks under time-invariant and time-varying topological perturbations," IEEE Trans. Automat. Contr., vol. 66, no. 3, pp. 13751382, 2021.

[14] S. S. Mousavi, M. Haeri, and M. Mesbahi, "Laplacian dynamics on cographs: Controllability analysis through joins and unions," IEEE Trans. Automat. Contr., vol. 66, no. 3, pp. 1383-1390, 2021.

[15] Y. Zheng, J. Wang, and K. Li, "Smoothing traffic flow via control of autonomous vehicles," IEEE Internet of Things J., 2020.

[16] J. Wang, Y. Zheng, Q. Xu, J. Wang, and K. Li, "Controllability analysis and optimal controller synthesis of mixed traffic systems," in IEEE Intell. Vehicles Sympos., 2019, pp. 1041-1047.

[17] J. Wang, Y. Zheng, Q. Xu, J. Wang, and K. Li, "Controllability analysis and optimal control of mixed traffic flow with human-driven and autonomous vehicles," arXiv:2002.02099, 2020.

[18] I. G. Jin and G. Orosz, "Optimal control of connected vehicle systems with communication delay and driver reaction time," IEEE Trans. Intell. Transport. Syst., vol. 18, no. 8, pp. 2056-2070, 2016.

[19] E. Vinitsky, K. Parvate, A. Kreidieh, C. Wu, and A. Bayen, "Lagrangian control through deep-rl: Applications to bottleneck decongestion," in 21st Internat. Conf. Intell. Transport. Syst., 2018, pp. 759765.

[20] C. Wu, A. M. Bayen, and A. Mehta, "Stabilizing traffic with autonomous vehicles," in IEEE Internat. Conf. Rob. Automat., 2018, pp. $1-7$.

[21] M. Di Vaio, G. Fiengo, A. Petrillo, A. Salvi, S. Santini, and M. Tufo, "Cooperative shock waves mitigation in mixed traffic flow environment," IEEE Trans. Intell. Transport. Syst., 2019.

[22] M. R. Jovanović and N. K. Dhingra, "Controller architectures: Tradeoffs between performance and structure," Europ. J. Contr., vol. 30, pp. 76-91, 2016.

[23] S. E. Li, Y. Zheng, K. Li, Y. Wu, J. K. Hedrick, F. Gao, and H. Zhang, "Dynamical modeling and distributed control of connected and automated vehicles: Challenges and opportunities," IEEE Intell. Transport. Syst. Mag., vol. 9, no. 3, pp. 46-58, 2017.

[24] G. Orosz, "Connected cruise control: modelling, delay effects, and nonlinear behaviour," Veh. Syst. Dyn., vol. 54, no. 8, pp. 1147-1176, 2016.

[25] M. Treiber, A. Hennecke, and D. Helbing, "Congested traffic states in empirical observations and microscopic simulations," Phys. Rev. E, vol. 62, no. 2, p. 1805, 2000.

[26] C. Scherer, P. Gahinet, and M. Chilali, "Multiobjective outputfeedback control via lmi optimization," IEEE Trans. Automat. Contr., vol. 42, no. 7, pp. 896-911, 1997.

[27] F. M. Callier and C. A. Desoer, Linear system theory. Springer Science \& Business Media, 2012.

[28] E. D. Sontag, Mathematical Control Theory: Deterministic Finite Dimensional Systems. New York: Springer Verlag, 1998.

[29] S. Boyd, L. El Ghaoui, E. Feron, and V. Balakrishnan, Linear Matrix Inequalities in System and Control Theory. Philadelphia, PA: Siam, 1994, vol. 15. 